Methods This paper shows that the process involved development of mini-CEX check list the training consistent internally of, The workshops included an introduction presentation, a mock test using same video exercise, application of Interactive Response System for discussions, a pilot study was started after the clinical preceptors had become familiar with how mini-CEX works.

Result The teaching method included teaching goals, content, strategies, and evaluation. Findings support the efficacy of improving nursing competency using teaching courses infused with core nursing values and enhanced through mini-CEX based teaching.

Conclusion The paper findings illustrate that the mini-CEX teaching and evaluation tool can help clinical preceptors to apply technical knowledge, increase clinical preceptors confidence, and ensure consistency in the performance of the technique.

\title{
REFERENCES
}

1 Al Ansari A, Ali SK, Donnon T. The construct and criterion validity of the mini-CEX: A meta-analysis of the published research. Academic Medicine 2013;88:413-20.

2 Cook DA, Beckman TJ, Mandrekar JN, et al. Internal structure of mini-CEX scores for internal medicine residents: Factor analysis and generalizability. Advances in Health Science Education 2010;15:633-45.

\section{THE EXPERIENCE OF MINI-CLINICAL EVALUATION EXERCISE(MINI-CEX) OF NURSING EDUCATION FOR CLINICAL PRECEPTORS}

Su-Ya Huang. Nursing, Chi Mei Medical Center, Tainan, Taiwan, Province of China

10.1136/bmjopen-2015-forum2015abstracts.89

Background The mini-Clinical Evaluation Exercise(mini-CEX) is a combined teaching and assessment tool, that include seven items of interaction between nurses and the patient. It is predominately used assess nurses' medical interview skills, physical examination skills, procedural skills, counseling skills, clinical judgment, organizational efficiency, and humanistic qualities/ professionalism, then feedback to the nurse is given by the clinical preceptors.

Objectives The purpose of this study is to describe the experiences of mini-CEX of nursing education for clinical preceptors to elevate performance for training and learning. 